\title{
Somatosensory neuron types identified by high-coverage single-cell RNA-sequencing and functional heterogeneity
}

\author{
Chang-lin $\mathrm{Li}^{1,{ }^{1,}}$, Kai-Cheng $\mathrm{Li}^{1,{ }^{1}}$, Dan $\mathrm{Wu}^{1}$, Yan Chen ${ }^{1}$, Hao Luo ${ }^{4}$, Jing-Rong Zhao ${ }^{1}$, Sa-Shuang Wang ${ }^{4}$, \\ Ming-Ming Sun ${ }^{2}$, Ying-Jin Lu ${ }^{1}$, Yan-Qing Zhong ${ }^{1}, \mathrm{Xu}-\mathrm{Ye} \mathrm{Hu}^{5}$, Rui Hou ${ }^{2}$, Bei-Bei Zhou ${ }^{2}$, Lan Bao ${ }^{3,4}$, \\ Hua-Sheng $\mathrm{Xiao}^{2}$, Xu Zhang ${ }^{1,4}$
}

${ }^{I}$ Institute of Neuroscience and State Key Laboratory of Neuroscience, CAS Center for Excellence in Brain Science, Shanghai Institutes for Biological Sciences, Chinese Academy of Sciences, Shanghai 20031, China; ${ }^{2}$ National Engineering Center for Biochip at Shanghai, Shanghai, China; ${ }^{3}$ State Key Laboratory of Cell Biology, Institute of Biochemistry and Cell Biology, Shanghai Institutes for Biological Sciences, Chinese Academy of Sciences; ${ }^{4}$ School of Life Science and Technology, ShanghaiTec University, Shanghai 200031, China, ${ }^{5}$ Shanghai Clinical Center, Chinese Academy of Sciences/XuHui Central Hospital, Shanghai, China

Cell Research (2016) 26:967. doi:10.1038/cr.2016.90; published online on 2 Aug 2016

Correction to: Cell Research (2016) 26:83-102. doi:10.1038/cr.2015.149; published online on 22 December 2015

There is a labeled error in Supplementary information, Figure $\mathrm{S} 5 \mathrm{E}$ in the initial published version of this article. The labeling in this panel "S5-1", "S5-2", "S61 " and "S6-2" should be corrected into "C5-1", "C52", "C6-1" and "C6-2". The changes do not alter any of the conclusions drawn from this study. The corrected Supplementary information, Figure S5 is replaced in the online version of this article. The authors sincerely regret these errors and apologize for any inconvenience that they may have caused. 